\title{
RELAÇÃO ENTRE A AUTOPERCEPÇÃO DO ESTADO DE SAÚDE E A AUTOMEDICAÇÃO ENTRE ESTUDANTES UNIVERSITÁRIOS
}

\author{
RELATION BETWEEN SELF-PERCEIVED HEALTH STATUS AND \\ SELF-MEDICATION AMONG COLLEGE STUDENTS
}

\begin{abstract}
RESUMO I Objetivo: Relacionar criticamente a autopercepção acerca do estado de saúde e doença e a prática de automedicação entre estudantes de um curso de saúde. Metodologia: Utilizou-se um questionário semi-estruturado sobre as concepções e práticas ligadas à saúde e doença entre estudantes do Bacharelado Interdisciplinar em Saúde, na cidade de Salvador, Bahia. Foram analisadas as respostas sobre a percepção da saúde e a referência à adoção da automedicação. Resultados: Responderam ao questionário 503 estudantes, dentre os quais $82 \%$ se perceberam como saudáveis e $18 \%$ referiram a prática da automedicação quando doentes. Apenas $9,5 \%$ reportaram se sentir doentes, enquanto $88 \%$ afirmaram não se sentir assim. Entre os que se percebiam como doentes, houve significativamente uma maior frequência de referência à automedicação $(30 \%$ versus $16 \%)(p=0,04)$. Conclusão: $O$ estudo sugere uma relação entre a autopercepção do estado de saúde e a automedicação como prática adotada, entre os pesquisados. $O$ baixo percentual de automedicação encontrado nesta amostra de ingressantes, quando comparado a estudos com indivíduos em processo mais avançado de formação profissional, pode servir como um alerta aos cursos de saúde, apontando para a própria caminhada dentro do curso como um elemento que influencia a prática da automedicação.
\end{abstract}

Maria Thereza Ávila Dantas Coelho', Vanessa Prado dos Santos², Maria Beatriz Barreto do Carmo ${ }^{3}$, Adailton Conceição de Souza ${ }^{4}$, Carolina Pereira Xavier França ${ }^{5}$

Autora para correspondência: Maria Beatriz Barreto do Carmo - therezacoelho@gmail.com 'Doutora em Saúde Coletiva. Professora na Universidade Federal da Bahia (UFBA). Salvador, Bahia, Brasil.

${ }^{2}$ Doutora em Medicina. Professora na Universidade Federal da Bahia (UFBA). Salvador, Bahia, Brasil.

${ }^{3}$ Doutora em Ciências. Professora na Universidade Federal da Bahia (UFBA). Salvador, Bahia, Brasil.

${ }^{4}$ Mestre em Estudos Interdisciplinares sobre a Universidade. Professor da Rede FTC, Unijorge e Municipal de Ensino de Salvador. Salvador, Bahia, Brasil. ${ }^{5}$ Bacharelado Interdisciplinar em Saúde em andamento na Universidade Federal da Bahia (UFBA). Bolsista de Iniciação Científica PIBIC/CNPQ. Salvador, Bahia, Brasil.

Palavras-chave: saúde, doença, automedicação, educação superior.

\begin{abstract}
Objectives: Find out if there is relationship between self-perception of health and illness and the practice of self-medication among college students. Methods: We applied a questionnaire about the concepts and practices of health and illness among students of the Interdisciplinary Bachelor's Degree in Health, in the city of Salvador, Bahia. We analyzed the responses to the perception of health and the reference to the adoption of self-medication. Results: Among the 503 students, 82\% self-reported as healthy and $18 \%$ used self- medication when they were sick. Only $9.5 \%$ of students reported feeling sick, while $88 \%$ said they did not feel sick. Among students who perceived themselves as sick, there was significantly greater frequency of references to self-medication $(30 \%$ versus $16 \%)(p=0.04)$. Conclusions: Our study suggests a relation between the perception of young people as sick and selfmedication. The low percentage of self-medication found in this sample, compared to studies with individuals in more advanced process of professional training, can serve as a warning to health courses, pointing to their own progress within the course as an element that influences the practice of self-medication.
\end{abstract}

Key words: health, sickness, self-medication, higher education. 


\section{INTRODUÇÃO}

Ao longo da história, foram sendo construídas diferentes teorias interpretativas sobre 0 processo saúde-doença, mediadas pelos conhecimentos disponíveis e pelo contexto cultural de cada época. Tais teorias revelam, sobretudo, determinadas formas de pensar o mundo, o ser humano e, por consequência, o fenômeno da saúde, e traduziramse em uma variedade de projetos filosóficos diversos, muitas vezes divergentes.

Em sua revisão, Scliar (2007) nos apresenta um panorama da diversidade de apreensões possíveis para a saúde e a doença, destacando-se alguns períodos históricos. Partindo da Antiguidade com a centralidade da noção de equilíbrio/desequilíbrio, seguindo até a Medicina Grega e a visão Hipocrática que se contrapunha ao olhar mágico-religioso sobre a saúde e a doença, posteriormente aportando na idade média européia, quando a influência da religião cristã fez recrudescer a concepção de doença como resultado do pecado e a cura como questão de fé, chegou até a transição entre os séculos XVII e XIX, período no qual a medicina científica se desenvolveu de forma vertiginosa, sob a égide do paradigma cartesiano, especialmente com o progresso da Anatomia, da Fisiologia e da Patologia, que legaram o sistema classificatório das doenças (Oliveira, 2000). Nesse ponto da história, ocorre o "deslocamento epistemológico" da medicina moderna, passando de uma "arte de curar indivíduos doentes para uma disciplina das doenças", e instaurando o ideal amplamente disseminado de saúde como ausência de doença, que atravessou todo o século XX (Luz, 1979).

Verificamos, assim, que cada sociedade constrói um sistema simbólico acerca do processo saúde-doença, em diálogo com os tempos históricos, que influencia a autopercepção sobre o estado de saúde, sendo esta última, ao menos em parte, uma construção sociocultural (JYLHA, 2009). Essa autopercepção se origina por meio de um processo cognitivo, que ocorre a partir da aquisição de informações, significados, interpretações e representações, que são adquiridos, segundo Jylha (2009), a partir do ambiente sociocultural a que $\circ$ indivíduo pertence. Ela se dá por meio do entendimento que o indivíduo tem acerca do que é saúde. Sendo assim, não existe uma definição universal desse termo.

$\mathrm{Na}$ sua definição do conceito de saúde, a Organização Mundial de saúde (Who, 1989) leva em consideração aspectos físicos, mentais e sociais do indivíduo. Na autopercepção sobre o estado de saúde, Bailis, Segall e Chipperfield (2003) mostram que as pessoas associam ter ou não saúde mais com a ausência ou presença de sintomas físicos. Entretanto, é reconhecido que os fatores sociais, como a educação e a distribuição de renda, por exemplo, influenciam nas condições de saúde do indivíduo (Mansyur, et al, 2008). Esses fatores socioeconômicos também são utilizados como base para avaliar a autopercepção da saúde (Jylha, 2009). Em países em desenvolvimento, onde há grandes diferenças regionais, as desigualdades sociais e econômicas podem originar diferentes percepções sobre $\circ$ estado de saúde entre os indivíduos (Reichert, Loch \& Capilheira, 2012). A autopercepção de saúde pode ainda ser alterada pelo surgimento de novas circunstâncias, como o início da prática de atividades físicas, através de um diagnóstico inesperado de uma doença crônica, ou por meio de uma mudança para ambientes onde há uso indiscriminado de tabaco, tudo isso promovendo no indivíduo uma reflexão sobre seu próprio estado de saúde (Reichert, Loch \& Capilheira, 2012).

A automedicação, por sua vez, como a conhecemos hoje, é uma prática ligada ao consumo de fármacos, sem prescrição ou orientação médica. No entanto, o uso de uma diversidade de recursos terapêuticos para o alívio da dor e em busca da cura, segundo Rey (1997), configura-se como uma prática que atravessa diversos períodos históricos, apresentando-se como um recurso leigo para o autocuidado.

O tema do autocuidado vem sendo abordado no campo da filosofia, transparecendo no conceito de 'cuidado de si mesmo', por exemplo, resgatado por Michel Foucault (2004) da Antiguidade Clássica, referindo- se a uma noção complexa utilizada pelos Gregos para caracterizar um conjunto de atitudes e práticas concernentes ao fato de ocupar- 
se e preocupar- se consigo mesmo. $O$ cuidado de si implicaria, portanto, uma responsabilização do sujeito consigo, pressupondo uma relação singular e ativa com a verdade e com o saber, bem como com a sua aplicação prática sobre a própria saúde (Bub, 2006).

Na contramão desse conceito e de sua intrínseca relação com a autonomia e auto-responsabilização, a medicina moderna caracteriza-se por um conjunto de elementos que, por sua vez, desresponsabiliza os sujeitos, tornando-os passivos e coadjuvantes do seu processo de cuidado, tutelados pelo poder do conhecimento científico que automatiza e padroniza as práticas de cuidado à saúde. Especificamente no que se refere à automedicação, objeto de interesse desta pesquisa, as atuais circunstâncias socioculturais, aliadas ao desenvolvimento da farmacoterapia em sua dimensão de mercado, conferem a essa prática um caráter especial na atualidade (Lopes, 2001, 2007). Constituindo-se como uma expressão da crescente medicalização e farmacologização das sociedades modernas, ela representa $\circ$ avanço do domínio médico sobre um número cada vez maior de problemas cotidianos, denominado de 'medicalização da vida'. A despeito dessa questão, é importante considerarmos que, no campo da educação, tem-se discutido sobre os processos de medicalização que transformam as grandes questões políticas, sociais, culturais e afetivas dos sujeitos em questões médicas, invadindo, pois, diversas áreas da vida social e produzindo uma epidemia de diagnósticos (Souza, 2014).

Como argumentou Lopes (2001), observamos uma incorporação cada vez maior, sobretudo nas gerações mais jovens, da familiaridade com os medicamentos, além de uma apropriação leiga dos critérios de prescrição médica, devido à crescente padronização dos mesmos. Podemos inferir, também, que o avanço da internet e seu uso amplo pela sociedade colaboram para a busca de informações e rotinas de prescrição de fármacos.

No Brasil em especial, o sistema de saúde e seu modelo assistencial hegemônico (médico-assistencialprivatista), ao ser eminentemente centrado no indivíduo e composto por práticas curativas e normativas, conforme Teixeira (1998), podem ser entendidos como aspectos que engendram e fortalecem as práticas de automedicação em nossa sociedade. $O$ cenário da automedicação na atualidade revela, adicionalmente, uma progressiva deslegitimação dos recursos leigos e tradicionais na 'gestão terapêutica do cotidiano', sendo os fármacos entidades terapêuticas sobre as quais o indivíduo leigo, por mais que conheça suas formas de uso, desconhece os seus processos de produção, ao contrário dos recursos terapêuticos tradicionais, produzidos no contexto dos espaços domésticos, nos quais os saberes sobre os usos e a produção apresentavam-se indissociáveis. Portanto, a automedicação apresenta-se como uma prática que expressa, em si mesma, a crescente dependência do indivíduo leigo do sistema médico formal (Teixeira, 2013). Compreendemos que a autopercepção do estado de saúde pode influenciar as relações sociais dos indivíduos e as suas atividades cotidianas, estando também associada às suas escolhas por determinadas práticas de cuidado à saúde, incluindo a automedicação. Deste modo, o objetivo do presente estudo foi investigar a relação entre a autopercepção do estado de saúde e doença, entre jovens universitários, e a prática de automedicação.

\section{MATERIAIS E MÉTODO}

Este estudo foi realizado com estudantes ingressantes no Bacharelado Interdisciplinar (BI) em Saúde da Universidade Federal da Bahia. Trata-se de um curso de graduação de caráter interdisciplinar, que visa uma formação geral humanística, científica e artística, somada ao aprofundamento no campo da saúde. $\mathrm{O} B \mathrm{Bl}$ em Saúde pode representar um primeiro ciclo de formação profissional na área da saúde, sendo que, após o seu término, o estudante pode ingressar em um dos cursos profissionais de graduação da área da saúde, como, por exemplo, medicina, enfermagem, fisioterapia, nutrição ou odontologia, dentre outros (Teixeira, 2013).

Foi aplicado um questionário, elaborado pelo grupo de pesquisa, aos estudantes do Bacharelado Interdisciplinar em Saúde. A amostra foi composta por estudantes recém ingressos no curso, sendo 123 ingressantes em 201 1, 185 em 2012 e 195 em 2013, 
num total de 503 participantes. $O$ questionário continha onze questões abertas e, dentre estas, três foram selecionadas para análise nesta etapa da pesquisa. As questões analisadas foram: "Você se sente saudávelę; Você se sente doente?; Que providências você toma quando está doente? As perguntas selecionadas para análise tiveram o objetivo de relacionar a autopercepção do estado de saúde/doença com a prática da automedicação e foram respondidas de forma livre e aberta, sem direcionamento prévio.

As respostas dos estudantes ao questionário foram digitadas e tabuladas em planilha. A análise foi realizada através do programa estatístico EPI-
INFO 2005. Dentre as respostas dos estudantes, as consideradas aqui foram as de SIM ou NÃO, a respeito da questão sobre a autopercepção quanto à saúde ou doença, relacionando-as à citação ou não da automedicação como prática adotada quando se encontravam doentes. Verificou-se também se houve diferença entre a citação ou não da prática da automedicação de acordo com o sexo e a idade dos estudantes. Utilizamos o teste do Qui-quadrado para efetuar a comparação entre as variáveis e consideramos significante um $p \leq 0,05$. $O$ estudo foi aprovado pelo Comitê de Ética em Pesquisa da Secretaria de Saúde da Bahia, através do ofício $067 / 2010$. Todos os participantes assinaram termo de consentimento livre e esclarecido.

\section{RESULTADOS}

Analisando as características da amostra, dentre os 503 estudantes, 354 (71\%) eram do sexo feminino, $48 \%$ havia cursado o ensino médio em instituição pública e a média de idade foi de 23 anos.

Em relação à autopercepção de saúde-doença, 499 discentes responderam à pergunta "você se sente saudável?", sendo que 408 estudantes (82\%) responderam 'sim', se sentiam saudáveis; 81 (16\%) referiram que não se sentiam saudáveis; e 10 estudantes $(2 \%)$ não responderam de forma direta à pergunta (Tabela 1). Perguntados se sentiam- se doentes, encontramos que 496 responderam à pergunta, sendo que $47(10 \%)$ referiram sentir-se doentes, 437 (88\%) afirmaram não se sentir doentes e $12(2 \%)$ não forneceram respostas precisas, do tipo sim/não, não sendo possível portanto a categorização destas 12 respostas.

Quando perguntados sobre que providências tomavam quando ficaram doentes, 422 estudantes $(85 \%)$ responderam que procuravam assistência médica e 88 (18\%) mencionaram a automedicação como prática adotada.

Tabela 1

Respostas dos estudantes quando perguntados sobre a sua autopercepção do estado de saúde/doença e as medidas adotadas quando doentes.

\begin{tabular}{|c|c|c|}
\hline \multirow{3}{*}{ Pergunta } & \multicolumn{2}{|c|}{ Respostas } \\
\hline & Sim & Não \\
\hline & $N(\%)$ & $N(\%)$ \\
\hline Você se sente saudávelę & $408(82 \%)$ & $81(16 \%)$ \\
\hline Você se sente doente? & $47(10 \%)$ & $437(88 \%)$ \\
\hline \multicolumn{3}{|c|}{ Que providências você toma quando fica } \\
\hline \multicolumn{3}{|l|}{ doente? } \\
\hline Referiu-se à atenção médica & $422(85 \%)$ & $73(15 \%)$ \\
\hline Referiu-se à automedicação & $88(18 \%)$ & $407(82 \%)$ \\
\hline
\end{tabular}


Comparamos a autopercepção do estado de saúde como 'saudável' entre os sexos e obtivemos que, entre os homens, $82 \%$ se sentiam saudáveis, sendo esta a mesma porcentagem entre as mulheres, não havendo diferença entre ambos os grupos $(p=0,37)$. Quanto à autopercepção como 'doentes', apenas $11 \%$ dos homens e $9 \%$ das mulheres responderam que se percebiam como doentes, também sem diferença significante entre os grupos $(p=0,48)$.

Comparando os sexos quanto à citação da 'automedicação' como prática/providência quando doentes, $16 \%$ dos homens citaram tal prática, o mesmo sendo informado por 19\% das mulheres $(p=0,23)$, diferença esta não estatisticamente significante. A média de idade dos estudantes que citaram a automedicação como prática adotada foi de 23 anos, e a dos estudantes que não citaram a automedicação também foi de 23 anos, não sendo esta diferença entre os grupos significativa $(p=0,9)$.
Foi observada a presença de associação entre a autopercepção do estado de saúde com a citação da automedicação como prática/ providência quando doentes. Entre os estudantes que se autopercebiam como saudáveis, 67 (17\%) citaram a automedicação como prática adotada; porcentagem semelhante aos discentes que não se autopercebiam como saudáveis $18(23 \%)(p=0,22)$. Já entre os estudantes que se autopercebiam como doentes, encontramos que 30\% (14 graduandos) citaram a automedicação como prática adotada; e entre aqueles que não se autopercebiam como doentes, a prática de automedicação foi referida por apenas 16\% (71 estudantes), havendo diferença estatisticamente significante entre os grupos $(p=0,04)$. A análise da associação entre a autopercepção do estado de saúde/doença e a citação da prática da automedicação se encontra detalhada na tabela 2.

Tabela 2

Correlação entre a autopercepção do estado de saúde/doença dos estudantes e a referência à automedicação como prática adotada quando doentes.

\begin{tabular}{lcc}
\hline \multirow{2}{*}{ Quanto à autopercepção } & \multicolumn{2}{c}{ Citou a prática da Automedicação } \\
\cline { 2 - 3 } & Sim & Não \\
\cline { 2 - 3 } & $N(\%)$ & \\
\hline Você se sente saudávelẹ $(p=0,22)$ & $67(17 \%)$ & $339(83 \%)$ \\
Sim & $18(23 \%)$ & $60(77 \%)$ \\
Não & & $32(70 \%)$ \\
Você se sente doente? $(p=0,04)$ & $14(30 \%)$ & $365(84 \%)$ \\
Sim & $71(16 \%)$ & \\
Não &
\end{tabular}

\section{DISCUSSÃO}

A automedicação consiste no uso de medicamentos sem prescrição e acompanhamento médico e é considerada pela Organização Mundial da Saúde como uma prática de autocuidado, desde que feita de forma segura e racional (ANVISA, 2010; $W H O, 1989)$. Os motivos que mais contribuem para a prática da automedicação são: influência de propagandas, prescrições antigas, influência de funcionários da farmácia, amigos e vizinhos. Um estudo realizado por Galato, Madalena e Pereira (2012) mostrou que a dor é um dos problemas de saúde que mais leva a essa prática e que os medicamentos mais procurados são os analgésicos e os antitérmicos.

A ausência de fiscalização nas propagandas, bem como nas vendas de medicamentos, é um fator 
que pode estimular a automedicação. No Brasil, com o objetivo de regular a publicidade sobre o medicamento, foi publicada a Resolução Diretoria Colegiada, de 30 de Novembro 102/2000 (ANVISA, 2000). Nos resultados parciais da primeira etapa do Projeto de Monitoramento de Medicamentos, elaborado pela Anvisa (2000), foi observado o descumprimento dessa respectiva lei, considerando que um grande número de propagandas sugere ausência de efeitos colaterais nos medicamentos.

Em relação ao contexto específico da educação, ○ ideário que rege o cotidiano das instituições educacionais é também marcado pela disseminação epidêmica de supostos transtornos, fazendo com que $\circ$ cenário submeta-se à lógica da medicina (Collares \& Moysés, 2014). Embora esse processo já venha ocorrendo há algum tempo, observa-se um crescimento na última década, o que reflete também a própria mercantilização da vida em diversos âmbitos. O discurso contemporâneo, que tem na mídia um de seus principais aliados, é marcado pela lógica da biologização da vida, sendo que o discurso social está povoado de enunciados da ciência em sua perspectiva medicalizante. Proliferam-se, então, simplificações e mitificações que produzem efeitos nos sujeitos (Guarido, 2011).

É importante destacar que o medicamento possui significados que são construídos socialmente. Para entendê-los, é necessário primeiro pensar no significado de saúde. Lefèvre (1987) afirma que saúde está associada a um bem de consumo, sendo - medicamento um meio para obtê-la. A saúde ganha sentido quando surgem situações que geram desconforto, como a dor, fraqueza e ameaça à morte, por exemplo. Ela passa a existir dentro da sociedade de mercado, onde o medicamento surge como solução para situações nocivas (Jylha, 2009). A partir da compreensão de que existe relação entre a autopercepção do estado de saúde e a prática da automedicação, analisamos os resultados obtidos na nossa pesquisa. Na nossa amostra, a maioria dos alunos afirmou que se sente saudável, o que pode ser atribuído ao fato da maioria ser composta por jovens em torno dos vinte anos de idade. Pode-se inferir que os jovens avaliam seu estado de saúde a partir de terem ou não alguma doença grave e, uma vez que não a possuam, o estado de saúde é positivamente avaliado (Alves \& Rodrigues, 2005).
Alves e Rodrigues (2005) realizaram um estudo com o objetivo de analisar os determinantes da autopercepção da saúde entre idosos, em um Município de São Paulo. Esses autores encontraram que a presença de doenças crônicas contribuiu para uma autopercepção da saúde como negativa, pois quanto maior a quantidade de doenças crônicas que este grupo possuía, maior o percentual de respostas considerando a saúde como ruim. O mesmo ocorreu em relação à idade: quanto mais idosos eram os indivíduos, maior foi a probabilidade de uma resposta negativa sobre o estado de saúde.

Reichert, Loch e Capilheira (2012) observaram resultado semelhante em um estudo que teve como objetivo analisar a prevalência da autopercepção da saúde como regular/ruim entre adolescentes, adultos e idosos, e os fatores associados a ela. Nesse estudo, constatou-se uma grande associação entre morbidades como diabetes, hipertensão, doença pulmonar, cardíaca e câncer, e a autopercepção de saúde entre adultos e idosos. Quanto maior o número de comorbidades, maior também a prevalência de autopercepção da saúde como regular/ruim.

Estudo realizado por Garbin, et al. (2009) com adolescentes (idade média de 15 anos) revelou que $46,5 \%$ avaliou seu estado de saúde como 'ótimo' e $44,1 \%$ como 'bom', compreendendo um percentual elevado de avaliação positiva. Os resultados observados entre idosos, quando comparados a populações mais jovens, podem revelar que a autopercepção sobre o estado de saúde relacionase também à perspectiva/expectativa de vida, sendo que idosos podem se julgar mais doentes quanto mais avançada for a idade, pela redução da perspectiva de continuar vivo. Entre os jovens, a própria juventude e perspectiva de muitos anos de vida, ainda que hipotética, pode interferir na autopercepção da saúde.

Analisando os resultados obtidos referentes ao sexo e à autopercepção de saúde e de doença, não houve diferença significativa. Já o estudo de Aquino, Barros e Silva (2010), realizado com estudantes de cursos como odontologia, enfermagem, fisioterapia, medicina, farmácia e nutrição, dentre outros da área da saúde, revelou um percentual maior do sexo feminino entre os que fizeram referência à prática da automedicação. 
A associação significativa entre a maior referência à prática da automedicação entre os alunos que se sentiam doentes é indicativa do valor simbólico do medicamento como instrumento para a recuperação da saúde. Dessa forma, o medicamento tornase um instrumento rápido para o alívio dos sintomas e desaparecimento da doença (Alves \& Rodrigues, 2005). Os alunos, em suas respostas, também associaram a procura pelo medicamento sem prescrição com o tratamento para sintomas e doenças consideradas por eles como de pouca gravidade.

Loyola, Costa e Uchôa (2004) realizaram um estudo com o objetivo de identificar elementos contextuais que inibiam ou influenciavam a prática da automedicação. Verificaram que existe uma separação entre problemas de saúde que podem ser solucionados sem prescrição e aqueles que não podem prescindir da mesma. Essa distinção é feita a partir da gravidade atribuída à doença. Nesse sentido, pelo fato de a maioria dos nossos estudantes serem jovens e não estarem na faixa etária na qual é comum o acometimento de doenças graves, isso pode influenciar na escolha em se automedicar a partir da gravidade da doença. A dificuldade de acesso aos serviços de atenção, associada à imagem de saúde relacionada à juventude, também pode contribuir com essa prática, mas chama a atenção a importância dos aspectos subjetivos, especificamente a autopercepção, influenciando a adoção de determinadas práticas em saúde.

Relacionando $\circ$ nosso trabalho com os resultados obtidos em outros estudos, verificou-se que uma porcentagem reduzida de alunos citou a prática da automedicação ( $18 \%$ do total de 503 alunos). Um estudo realizado com o objetivo de verificar a automedicação entre 89 estudantes de enfermagem, associada a problemas de saúde, mostrou que $65,17 \%$ adotavam essa prática.

Aquino, Barros e Silva (2010) também realizaram um estudo com o objetivo de verificar o comportamento de estudantes da área de saúde e a prática de automedicação, considerando os últimos 15 dias que antecederam a aplicação do questionário. Nesse estudo, participaram um total de 223 estudantes e, deste total, $57,7 \%$ utilizaram medicamento sem prescrição médica. Figueiredo et al. (2009), com o objetivo de verificar o comportamento de estudantes do curso de medicina em relação à prática da automedicação para perda de peso, aplicaram um questionário para 275 estudantes, no qual 55\% relataram ter se automedicado.

Algumas hipóteses foram consideradas para compreender a importante diferença do percentual de automedicação referida na nossa amostra, quando comparada aos estudos supracitados. Como na presente pesquisa a pergunta sobre automedicação não foi colocada de forma direta, sendo apenas solicitado que os estudantes mencionassem as práticas adotadas quando doentes, não indicando previamente nenhuma delas em particular, pode ter ocorrido uma sub-referência desta prática, seja por viés de memória ou mesmo por não entenderem a automedicação como uma opção viável de resposta.

Outro aspecto importante a ser considerado devese ao fato de que, no nosso estudo, a amostra foi composta por jovens recém ingressos na universidade. Isso denota uma diferença importante dos estudos citados acima, que se referem a estudantes universitários em etapas profissionalizantes e com mais tempo no curso de graduação (Santos et al, 2012; Figueiredo et al, 2009). Ou seja, é possível que a própria formação na área profissional da saúde, a exemplo dos cursos de medicina, fisioterapia e odontologia, dentre outros ligados às amostras dos estudos citados, seja, por seu caráter biomédico intrínseco, produtora de um discurso medicalizante e que, por sua vez, influencie os discentes na prática da automedicação. Além disso, o conhecimento técnico e científico acerca de patologias e a familiaridade com suas terapêuticas, da parte dos estudantes, podem também influenciar e favorecer tal prática.

\section{CONSIDERAÇÕES FINAIS}

No nosso estudo, a maioria dos estudantes se autopercebeu como saudável. Foi também observado um baixo percentual de estudantes que citaram a automedicação como prática de cuidado. Por outro lado, os jovens universitários que se 
percebiam como doentes citaram mais a prática da automedicação, o que sugere que a autopercepção do estado de saúde influencia as escolhas e práticas de autocuidado. Considerando que a ausência de pergunta direta sobre a automedicação pode ter gerado uma menor referência a esta prática na nossa amostra, pretende-se, para as próximas etapas do estudo, incluir uma questão específica sobre práticas de cuidado à saúde que inclua a automedicação como uma das opções de resposta. Em relação ao baixo percentual de automedicação encontrado na amostra em geral, esse dado pode servir como um alerta para os cursos de saúde, pois as pesquisas com os graduandos em estágios mais avançados de suas formações revelam, por sua vez, um percentual bem maior de automedicação, apontando para a própria caminhada dentro do curso como um elemento que influencia esta prática.

Portanto, algumas questões se colocam como pertinentes aos cursos da saúde, como, por exemplo, de que modo pode-se discutir a automedicação no âmbito desses cursos? Como promover sujeitos e futuros profissionais da saúde que pratiquem $\circ$ autocuidado de forma ampliada e que, no caso de fazerem uso da automedicação, o façam de forma segura e responsável? Tais aspectos apontam para a importância do incremento de currículos e práticas pedagógicas interdisciplinares nos cursos superiores da área, incluindo outros referenciais epistemológicos na abordagem da saúde, para além do modelo biomédico hegemônico. Estudos posteriores podem contribuir para o aprofundamento dessas questões.

\section{CONFLITOS DE INTERESSES}

Nenhum conflito financeiro, legal ou político envolvendo terceiros (governo, empresas e fundações privadas, etc.) foi declarado para nenhum aspecto do trabalho submetido (incluindo mas não limitandose a subvenções e financiamentos, conselho consultivo, desenho de estudo, preparação de manuscrito, análise estatística, etc).

\section{REFERÊNCIAS}

Agência Nacional de Vigilância Sanitária - ANVISA. (2010). Projeto de Monitoração de Propaganda e Publicidade de Medicamentos - Fase I (2002 - 2003). Brasília: ANVISA
Alves, L., Rodrigues, R.N. (2005). Determinantes da autopercepção de saúde entre idosos do Município de São Paulo, Brasil. Rev Panam Salud Publica, 5(17), 333341. doi: 10.1590/S1020-49892005000500005

Aquino, D.S., Barros, J.A.C, Silva, M.D.P. (2010). A automedicação e os acadêmicos da área da saúde. Ciênc. saúde coletiva, 5(15): 2533-2538. doi: 10.1590/S141381232010000500027

Bailis, D.S., Segall, A., Chipperfield, J.G. (2003). Two views of self-rated general health status. Social Science \& Medicine, 2(56), 203-217

Bub, M.B.C., Medrano, C., Silva, C.D., Wink, S., Liss, P.E., Santos, E.K.A. (2006). A noção de cuidado de si mesmo e o conceito de autocuidado na enfermagem. Texto contexto - enferm., 15(spe): 152-157. doi: 10.1590/s010407072006000500018

Collares, C.A.L., Moysés, M.A.A.A. (2014). Educação na era dos transtornos. In: Viégas LS, Ribeiro MIS, Oliveira EC, Teles LAL. Medicalização da educação e da sociedade: ciência ou mito?, (pp.21-43). Salvador: EDUFBA.

Figueiredo, E.T., Morais, A.M., Costa, A.M.D.D., Terra, F.S. (2009). Perfil dos estudantes de Medicina frente à automedicação para redução de peso. Rev Bras Clin Med., 6(7), 385-389

Foucault, M. (2004). Hermenêutica do sujeito. São Paulo: Martins Fontes

Galato, D., Madalena, J., Pereira, G.B. (2012). Automedicação em estudantes universitários: influência da área de formação. Ciênc. Saúde Coletiva, 12(17): 3323-3330. doi: $10.1590 / \mathrm{S} 1413-81232012001200017$

Garbin, C.A.S, Garbin, A.J.I., Moimaz, S.A.S, Gonçalves, P.E. (2009). A saúde na percepção do adolescente. Physis, 1(19), 227-238. doi: 10.1590/S010373312009000100012

Guarido, R.A. (201 1). Biologização da vida e algumas implicações do discurso médico sobre a educação. In: Conselho Regional de Psicologia de São Paulo. Medicalização de crianças e adolescentes: conflitos silenciados pela redução de questões sociais a doenças de indivíduos, (pp.27-39). São Paulo: Casa do Psicólogo.

Jylha, M. (2009). What is self-rated health and why does it predict mortality? Towards a unified conceptual model. Social Science \& Medicine, 3(69), 307-316. doi: 10.1016/i.socscimed.2009.05.013

Lefèvre, F. (1987). A oferta e a procura de saúde através do medicamento: proposta de um campo de pesquisa. Rev. Saúde Pública, 1(21), 64-67. doi: 10.1590/S003489101987000100010

Lopes, N. (2001). Automedicação: algumas reflexões sociológicas. Sociologia, problemas e práticas, (37), 141 165 
Lopes, N. (2007). Automedicação, saberes e racionalidades leigas em mudança. Revista Crítica de Ciências Sociais, $78,119-138$

Loyola, A.I., Costa, M.F.L., Uchôa, E. (2004). Projeto Bambuí: uma abordagem qualitativa na investigação da automedicação. Cad. Saúde Pública. 20(6), 1661-1669. doi: $10.1590 /$ S0102-311X2004000600025

Luz, M.T. (1979). As instituições médicas no Brasil: instituição e estratégia de hegemonia. Rio de Janeiro: Graal

Mansyur, C., Amick, B.C., Harrist, R.B., Franzini, L. (2008). Social capital, income inequality, and self-rated health in 45 countries. Social Science \& Medicine, 66(1), 43-56. doi: 10.1016/i.socscimed.2007.08.015

Oliveira, M.A.C., Egry, E. (2000). A historicidade das teorias interpretativas do processo saúde-doença. Rev. Esc. Enf., $34(1), 9-15$

Reichert, F.F., Loch, M.R., Capilheira, M.F. (2012). Autopercepção de saúde em adolescentes, adultos e idosos. Ciênc. Saúde Coletiva, 17(12), 3353-3362. doi: 10.1590/S141381232012001200020

ANVISA. Resolução RDC n 102 (30, novembro 2000). Aprova o regulamento sobre propagandas, mensagens publicitárias e promocionais de medicamentos. Brasília: ANVISA; 2000. Recuperado em http://www.febrafar.com.br/upload/ up_images $/$ rdc\%20102.pdf

Rey, L. (1997). Dicionário de termos técnicos de medicamentos de medicina e saúde. Rio de Janeiro: Guanabara Koogan S.A

Santos, B., Souza, L.G, Delgado, N.M, Torres, W.O. (2012). Incidência da automedicação em estudantes de Enfermagem. J. Health Sci Inst., 30(2), 156-160

Scliar, M. (2007). História do Conceito de Saúde. Rev. Saúde Coletiva, 17(1), 29-41. doi: 10.1590/S010373312007000100003

Souza, B.P. (2014). Orientação à queixa escolar: na contramão da medicalização da educação e da vida. In: Viègas L.S., Ribeiro, M.I.S, Oliveira, E.C., Teles, L.A.L. Medicalização da educação e da sociedade: ciência ou mito?, (pp. 6990). Salvador, BA: EDUFBA

Teixeira, C.F., Paim, J., Vilasbôas, A.L. (1998). SUS, modelos assistenciais e vigilância da saúde. Informe Epidemiológico do Sus, 7(2), 7-28. doi: 10.5123/S010416731998000200002

Teixeira, C.F., Coelho, M.T.A.D., Rocha, M.N.D. (2013). Bacharelado Interdisciplinar: Uma proposta Inovadora na educação superior em saúde no Brasil. Ciênc. Saúde Coletiva, 18(6), 1635-1646. doi: 10.1590/S141381232013000600015

Who (1989). Guidelines for the Regulatory Assessment of Medicinal Products use in self-medication. Geneva. Recuperado de http://apps.who.int/medicinedocs/pdf/ s2218e/s2218e.pdf 